\title{
LES ALOSA FOSSILES ET LA NOTION DE GENRE EN SYSTÉMATIQUE PHYLOGÉNÉTIQUE.
}

\section{R. ZARAGÜETA BAGILS}

Laboratoire de Paléontologie, UMR 8569, Institut de Systématique CNRS FR 1541, Muséum national d'Histoire naturelle, 8 rue Buffon, 75005 PARIS, France.

\section{RÉSUMÉ}

Un total de douze espèces fossiles a été attribué au genre Alosa. Une seule espèce provient de gisements oligocènes de Russie alors que les autres sont des espèces miocènes en provenance de Turquie ( 5 espèces), de l'Afrique du Nord (Oran, 4 espèces), du Caucase ( 1 espèce) et d'Allemagne ( 1 espèce).

La plupart de ces espèces seront retirées du genre lorsque les relations de parenté entre elles seront analysées. Elles n'appartiennent probablement pas au genre Alosa.

Mais, comment peut-on décider de l'appartenance d'un poisson, fossile ou actuel, à un genre? Alosa est un taxon d'un rang taxonomique. Pour la systématique évolutionniste, un taxon n'est rien d'autre qu'une classe, c'est-à-dire un regroupement d'objets partageant au moins l'un des caractères diagnostiques du groupe. En systématique phylogénétique, au contraire, un genre est un individu. II doit donc posséder primitivement l'ensemble des caractères diagnostiques et il possède une histoire propre. II peut comprendre un nombre arbitraire d'espèces et avoir une extension temporelle et spatiale quelconque. Par ailleurs, l'utilisation des catégories taxonomiques ne se justifie ni par la théorie de l'évolution (une théorie des mécanismes et des processus) ni par les méthodes de classification phylogénétique (ayant trait à la structure des relations de parenté). II s'agit plutôt d'une ingérence de la nomenclature dans la théorie générale des classifications.

En ce qui concerne les aloses, en l'absence d'étude phylogénétique, on ne peut pas se prononcer sur la pertinence de ce taxon. Les espèces actuelles partagent certains caractères de l'appareil branchial. Mais ces caractères ne sont pas observables chez les fossiles attribués au genre Alosa. Ils doivent être considérés, par conséquent, comme des Clupeidae incertae sedis.

\section{THE FOSSIL ALOSA AND THE CONCEPT OF GENUS IN SYSTEMATIC PHYLOGENY.}

\section{ABSTRACT}

Twelve fossil species have been assigned to the genus Alosa. One of them comes from the Oligocene of Russia, the other species are Miocene aged, 5 from Turkey, 4 from Oran (Algeria) one from the Caucasus Mountains and one from Germany. 
Probably most of these fossils will be removed from the genus when the interrelationships of the clupeid subgroups will be established. However, which are the criteria to decide if a fish, extant or fossil, belongs to a genus ?

Alosa is a taxon and the genus is a taxonomic level. For the evolutionary school of systematics, a taxon is just a class, i.e. a grouping of objects that share, at least, one of their characteristics. In phylogenetic systematics, a taxon is an individual. It must share, primitively, all of the diagnostic characters of the group and has its own history. It may group an arbitrary number of species that have no particular distribution in time and space. The use of taxonomic levels is neither justified by a theory of evolution (a theory of process an mechanisms) nor by the methods of phylogenetic classifications.

In the case of shads, without a phylogenetic study, nothing can be said about the phylogenetic validity of the genus. Recent species share certain characters of the gills, but these are not preserved in fossils "shads ". The fossil species assigned to Alosa have to be considered, as a consequence, as Clupeidae incertae sedis.

\section{INTRODUCTION}

Un total de douze espèces fossiles a été attribué au genre Alosa (GRANDE, 1985). Elles proviennent de gisements marins oligocènes ( 1 espèce de Russie) et miocènes ( 5 de Turquie, 4 d'Oran, une du Caucase et une d'Allemagne, Tableau I).

\section{Tableau I}

Liste des espèces fossiles de Clupeidae attribuées au genre Alosa.

\section{Table I}

List of Clupeidae fossil species assigned to the genus Alosa.

\begin{tabular}{|c|c|c|c|}
\hline Espèce & Age & Localité & Commentaires \\
\hline Alosa aralensis Chisara, 1977 & Oligocène & Russie & \\
\hline Alosa baykalii Rückert-Ulkümen, 1965 & Miocène & Turquie & \\
\hline Alosa crassa Sauvage, 1873 & Miocène & $\begin{array}{l}\text { Oran et } \\
\text { Italie }\end{array}$ & $\begin{array}{l}\text { Synonyme d'A. numidica d'après } \\
\text { WOODWARD (1901, p.151) }\end{array}$ \\
\hline Alosa elongata Agassiz, 1843 & Miocène & Oran & $\begin{array}{l}\text { Synonyme d'A. numidica d'après } \\
\text { WOODWARD (1901, p.151) }\end{array}$ \\
\hline Alosa fortipinnata Rückert-Ulkümen, 1965 & Miocène & Turquie & \\
\hline Alosa genuina Danil'chenko, 1960 & Miocène & Caucase & \\
\hline Alosa numidica Sauvage, 1873 & Miocène & Oran & $\begin{array}{l}\text { Synonyme d'A. elongata d'après } \\
\text { ARAMBOURG }(1927, \text { pl. } 18)\end{array}$ \\
\hline Alosa ovalis Rückert-Ulkümen, 1965 & Miocène & Turquie & \\
\hline $\begin{array}{l}\text { Alosa pinarhisarensis Rückert-Ulkümen, } \\
1965\end{array}$ & Miocène & Turquie & \\
\hline Alosa renoui Sauvage, 1873 & Miocène & Oran & $\begin{array}{c}\text { Synonyme d'A. numidica d'après } \\
\text { WOODWARD (1901, p.152) et } \\
\text { d'A. elongata d'après ARAMBOURG } \\
(1927, \text { pl. } 18)\end{array}$ \\
\hline Alosa sculptata (Weiler, 1920) & Miocène & Allemagne & \\
\hline Alosa weileri Rückert-Ulkümen, 1960 & Miocène & Turquie & \\
\hline
\end{tabular}


En dehors de leur description originale, aucun de ces taxons n'a fait l'objet d'autres recherches. Or, d'après GRANDE (1985), la plupart de ces espèces seront attribuées à d'autres genres lorsque les relations de parenté entre les Alosinae, les Clupeinae et les Dorossomatinae seront mieux comprises. En effet, l'analyse phylogénétique de GRANDE (1985), bien que basée sur peu de caractères, n'a pas pu établir la monophylie des sousgroupes des Clupeidae (les Alosinae, les Clupeinae, les Dorossomatinae, les Pellonulinae et les Dussumierinae). Si ces espèces ont été attribuées au genre Alosa, et si cette attribution peut être mise en doute à cause d'une éventuelle non-monophylie du genre, on peut se demander quels sont les critères pour décider de l'appartenance ou non d'une espèce à un genre. En définitive, la question qui est posée est de savoir ce qu'est un genre.

\section{Diagnoses et phylogénie}

Les systématiciens néodarwiniens ont toujours établi leurs classifications selon le regroupement en grades. Les grades sont des classes, c'est à dire que l'on n'exige pas des entités qui appartiennent à ces groupes la présence d'un ensemble complet de caractères diagnostiques, mais que certains d'entre eux, différents pour chaque espèce, soient jugés suffisants d'après l'expertise d'un systématicien (BEATY, 1982).

Les diagnoses d'espèce correspondent bien à cette idée. Or le but des diagnoses n'est pas de servir à inférer la phylogénie d'un groupe de taxons, mais de permettre leur identification et l'éventuelle attribution des populations nouvellement découvertes à un taxon décrit.

L'avènement de l'analyse phylogénétique ou cladistique, à partir notamment de la traduction anglaise de l'ouvrage de l'entomologiste allemand HENNIG (1966), a provoqué une révolution méthodologique en Systématique. La méthodologie proposée par cet auteur et les modifications qui y ont été apportées depuis permettent d'établir des classifications non pas de classes d'organismes mais d'individus (PATTERSON, 1982). Les organismes sont réduits à une liste de caractères présentant chacun au moins deux états, un état primitif (appelé plésiomorphe) et un état dérivé ou évolué (apomorphe). HENNIG a été le premier à comprendre que les états de caractères primitifs ne permettent pas le regroupement de plusieurs taxons. En effet, ils étaient déjà présents chez l'ancêtre du groupe et ne nous disent rien des relations de parenté entre ses descendants. Par exemple, la présence d'une carène ventrale, constituée par des écussons, ne nous permet pas de préciser l'histoire évolutive des Clupeiformes puisque l'ancêtre de ce groupe en possédait déjà une.

Or les systématiciens néodarwinens se sont servis de ces caractères pour regrouper des taxons. Les Clupeiformes ont été définis en fonction d'absences primitives, comme celles de l'appareil de Weber ou des épines aux nageoires; ou en fonction de présences tout aussi primitives comme les écailles cycloïdes, la position abdominale des nageoires pelviennes, etc. II n'y a pas si longtemps, tous les téléostéens basaux (Elopomorphes, Ostéoglossomorphes, Clupéomorphes, Ostariophyses anotophyses, Osmeriformes, Salmoniformes, Esocoïdes) étaient regroupés au sein d'un taxon Clupéiformes dont le contenu informatif était pratiquement nul (BERTIN et ARAMBOURG, 1958).

Pour savoir si un caractère est primitif ou évolué, c'est-à-dire pour le polariser afin d'enraciner l'arbre, deux critères sont utilisés. Le premier est la comparaison extra-groupe (= outgroup) (DARLU et TASSY, 1993), seul valable dans le cas des données moléculaires. II consiste à comparer l'état de caractère observé chez les taxons étudiés ('ingroup), avec celui des taxons externes mais proches et peu spécialisés (le ou les outgroups). C'est ce dernier qui sera considéré comme plésiomorphe. En effet, l'état primitif est l'état le plus généralisé, le moins spécialisé, en application des lois de 
VON BAER (1828), redéfines par NELSON (1978). En d'autres mots, l'état de caractère primitif est celui que l'on trouve dans l'outgroup et dans l'ingroup, alors que l'état dérivé n'est observé que dans l'ingroup (WILEY, 1976). Par exemple, les écussons ventraux sont un caractère dérivé des Clupeomorpha, puisqu'ils sont absents des groupes proches. L'absence est primitive dans ce cas. Retenons cependant qu'ils peuvent être secondairement perdus, et c'est alors la congruence avec l'ensemble de caractères qui le montrera (PATTERSON, 1982). Les groupes paraphylétiques n'ont pas d'existence historique. Ce ne sont pas des groupes naturels. Les seuls groupes naturels sont les groupes monophylétiques, c'est à dire les taxons constitués par un ancêtre et l'ensemble de ses descendants. Par exemple, les reptiles ou les poissons sont des groupes paraphylétiques. Ils ne comprennent que les taxons ayant conservé des caractères primitifs comme les écailles sur tout le corps ou les membres parasagitaux dans le cas des reptiles, les nageoires et les branchies dans le cas des poissons.

\section{La parcimonie}

La phase suivante consiste a en coder les différents états de caractère. Le symbole choisi pour le codage est parfaitement arbitraire. On a l'habitude d'utiliser les lettres standard (ATCG) s'il s'agit de nucléotides et des chiffres dans le cas de données morphologiques. Bien que, souvent, le codage 0 représente l'état primitif et $1,2 \ldots$ les états dérivés, il n'existe aucune contrainte méthodologique ou de la part des logiciels de traitement. On obtient ainsi une matrice (un tableau) qui sera traité, le plus souvent, par un logiciel de parcimonie (les données moléculaires peuvent aussi être traitées par la méthode du maximum de vraisemblance). Le logiciel va se charger d'optimiser les données selon le principe de parcimonie. Ce principe général établit que l'explication retenue sera celle qui minimise les hypothèses ad hoc pour expliquer un fait. Dans ce cas, le fait (l'observation) est constitué par la matrice de taxons / caractères. On fait une seule hypothèse, en imposant une structure hiérarchique pondérée (c'est à dire une structure d'arbre dans le sens de la théorie des graphes) aux données. La solution la plus parcimonieuse sera celle qui minimisera la longueur totale de l'arbre obtenu. Cette optimisation revient à maximiser l'homologie (ou minimiser l'homoplasie, somme des convergences et parallélismes).

Le traitement des données par parcimonie présente cependant un problème. II s'agit du temps nécessaire pour trouver l'arbre le plus parcimonieux. En effet, il s'agit d'un problème dit NP-complet (D'UDEKEM-GEVERS, 1990). Cette classe de problèmes présente une complexité algorithmique exponentielle, c'est à dire que l'ajout d'un taxon à la matrice va augmenter le temps de calcul d'une manière exponentielle. La limite pour que l'ordinateur trouve la bonne réponse après avoir exploré toutes les combinaisons possible est d'environ 20 taxons (le nombre de caractères n'a pratiquement pas d'influence). Au-delà de ce nombre de taxons terminaux, l'utilisation d'heuristiques s'impose. Ce type de recherche est extrêmement rapide mais ne garantit pas de trouver la bonne réponse (ce qui ne veut évidemment pas dire que la réponse fournit ne soit pas l'optimale). Si la solution n'est pas unique, c'est-à-dire s'il existe plusieurs arbres de longueur minimale, on calcule souvent l'arbre de consensus. II retient les clades qui apparaissent dans tous les arbres les plus parcimonieux.

Dans les cas de données morphologiques, l'analyse phylogénétique permet d'inférer les états de caractère aux nœuds, qui correspondent à l'ancêtre hypothétique du clade. En effet, l'analyse cladistique ne permet pas d'identifier un ancêtre parmi les taxons étudiés. II s'agit là d'une conséquence logique : l'ancêtre possédait tous les caractères dérivés du groupe (les caractères primitifs pour ces descendants) mais aucun caractère propre. II serait donc identifié comme appartenant au clade constitué par l'ensemble de ses descendants mais sa position systématique resterait irrésolue. Par exemple, le seul caractère dérivé potentiel du genre Alosa est la présence de l'encoche sur la mâchoire supérieure. Si le vrai ancêtre venait à être trouvé, le fossile posséderait l'encoche, 
synapomorphie du groupe. Ce caractère est donc dérivé pour le genre (par rapport aux non-aloses) mais primitif au sein du genre, donc inutile pour discuter des relations de parenté des aloses entre elles.

\section{Pattern et process, structure et processus}

Les tenants de la théorie synthétique ont soutenu qu'ils incluaient des renseignements sur l'écologie et la divergence morphologique, etc. Cependant ils n'ont jamais donné de critères permettant de quantifier ces données. De toute façon, la prise en compte de ces données n'a pas eu de conséquences sur la classification, restée fondamentalement inchangée depuis Linné. De plus, ces données ne font pas référence à la structure de la nature que les classifications prétendent reconstruire, mais aux processus (adaptation, anagenèse, relations avec l'environnement).

La faille des classifications synthétistes consiste en la confusion entre la structure de la nature (le pattern) et les processus qu'elle met en œuvre pour parvenir à cette structure. La systématique phylogénétique établit des classifications en fonction d'un critère explicite : la monophylie, définie à partir d'au moins une synapomorphie (caractère dérivé unique) et d'une seule hypothèse : la structure de la nature est hiérarchique, c'est à dire qu'elle équivaut à un emboîtement d'entités. Ainsi elle se propose de chercher la structure des relations de parenté, indépendamment des processus qui ont produit cette structure. Les classifications synthétiques, en prétendant inclure les données écologiques (importance de l'anagenèse, l'adaptation, l'utilisation de l'environnement), acceptent les groupes paraphylétiques. Le résultat en est une notable perte d'information (on ne sait pas si un groupe est monophylétique ou non) et la création de groupes souches à la définition vague et au contenu incertain. Les évolutionnistes néodarwiniens considèrent la phylogénie comme étant hors d'atteinte de la connaissance (ELDREDGE, 1985).

\section{La notion de genre en systématique phylogénétique}

Les catégories taxonomiques sont un héritage pré-évolutionniste des conceptions philosophiques aristotéliciennes. Les systématiciens évolutionnistes les ont incorporées à leur système en tant que quantificateur de la diversité morphologique, écologique, etc. Le Code de Nomenclature Zoologique définit la nomenclature comme étant indépendante de la taxonomie, l'une s'occupant de noms et l'autre de taxons, avec le type porte-nom pour seul lien. Or ce code ne respecte pas cette indépendance, et ceci pour plusieurs raisons, dont deux seront discutées ici.

D'abord, parce qu'il impose l'utilisation de catégories. Cela implique la reconnaissance des rangs taxonomiques, un concept non nomenclatural. Cet ouvrage, grâce à son rôle de référence propose et impose une seule vision de la taxonomie parmi toutes celles qui existent.

Le Code gère les noms du groupe-espèce, groupe-genre et groupe-famille, tout en laissant de côté le problème de l'espèce. On peut dire que les autres concepts appartiennent à l'une des écoles de Systématique, l'école néodarwinienne, encore appelée évolutionniste ou éclectique. Ni les tenants de la phénétique (et de ses méthodes de distances comme UPGMA et $\mathrm{NJ}$ ), ni ceux de l'école phylogénétique ou cladiste ne reconnaissent une réalité intrinsèque aux catégories taxonomiques.

Deuxièmement, le code ne s'occupe pas des catégories supérieures au groupe famille. Il existe néanmoins des règles de nomenclature quant à la dénomination de ces taxons. Il s'agit de l'utilisation de terminaisons identiques pour tous les taxons de même rang (-oidei, -iformes, etc.). Le concept de rang taxinomique est donc étroitement lié à l'activité nomenclaturale. Un auteur qui n'utilise pas les terminaisons conventionnelles 
(cela se fait de plus en plus pour certains groupes) affirme qu'il ne reconnaît pas les catégories.

Cette façon de faire est directement inspirée de la méthodologie cladiste. L'ingérence évidente de la nomenclature est la suivante: les terminaisons modifient la façon de penser. Ainsi, on compare un groupe dont la terminaison est « iformes » avec un groupe de même terminaison. Or, l'usage des terminaisons est tel qu'il reste pratiquement impossible de présenter une hiérarchie sans utiliser de catégories ce qui empêche la pleine expression de la conception cladiste de la classification. Bien que ces catégories ne soient pas gérées par le code de nomenclature, elles n'existent que parce que ce dernier admet l'existence du genre, de la famille et donc de toutes les autres catégories qui appartiennent à la même logique.

\section{Le genre Alosa}

Dans ce cadre conceptuel, quelle est la signification d'Alosa? En effet, les plus anciens fossiles attribués à ce genre apparaissent à l'Oligocène (LE GALL, 2000). Est-ce que cela veut dire que tous les genres de clupéidés sont " apparus » à l'Oligocène ou bien que la divergence morphologique entre les aloses et les Hilsha est plus grande qu'entre Diplomystus dentatus et $D$. birdi (deux clupéomorphes fossiles) ? En fait, les catégories taxonomiques ne nous apprennent rien quant à la vitesse d'évolution ou à la divergence des espèces.

En réalité, le rang taxonomique d'Alosa est parfaitement arbitraire et ne peut se justifier que pour des raisons pratiques. De plus, pour que ce soit un taxon naturel il faut définir au moins une synapomorphie. Les diagnoses des espèces fossiles, par exemple celles d'ARAMBOURG (1927) sur les Alosa du Miocène d'Oran sont une liste de caractères presque tous plésiomorphes ou variables. Cependant, comme pour les espèces actuelles, nous pouvons retenir un état de caractère évolué susceptible de nous informer sur l'histoire évolutive de ce sous-groupe de Clupéidés.

D'après WHITEHEAD (1985), les Alosinae se caractérisent par la présence d'une encoche de la mâchoire supérieure qui permet de loger la symphyse dentaire, le reste de caractères étant très variables parmi les Clupéidés. Le genre Alosa est caractérisé par la taille modérée à grande de ses membres, par la possession d'une carène ventrale saillante et par des caractères des branchispines.

L'encoche de la mâchoire est donc un caractère dérivé au sein des Clupéidés, mais il est primitif pour les aloses (il était présent sur le dernier ancêtre commun des Alosinae).

Les espèces classées dans le genre Hilsha se caractérisent, quant à elles, par la présence d'une striation des os du toit crânien. Sans une analyse phylogénétique prenant en compte plusieurs caractères, l'hypothèse de monophylie n'est pas testée, au sens poppérien. Par exemple, si la striation est apparue chez un membre apparenté aux aloses, alors Hilsha est un sous-groupe d'Alosa qui devient paraphylétique. Si, au contraire, la striation a été perdue chez les aloses à partir d'ancêtres où elle était présente, c'est Hilsha qui est un taxon paraphylétique, puisque diagnostiqué par un trait primitif, présent chez le dernier ancêtre commun aux aloses et aux membres d'Hilsha.

GRANDE (1985) pense que la plupart des espèces fossiles d'Alosa seront classées au sein d'un autre genre lorsque les relations de parenté entre les différents groupes de Clupéidés seront mieux comprises. A ce jour, il ne semble pas y avoir de synapomorphie connue pour aucun de ces groupes.

Dans l'état actuel des connaissances sur ces fossiles, cependant, leur attribution au genre Alosa peut être acceptée d'un point de vue nomenclatural mais non d'un point de 
vue systématique. Les noms des taxons ne sont pas seulement utilisés par des systématiciens, et leur aspect pratique pour des études de paléoécologie, faunistique, biostratigraphie et même commercial est un aspect important. Les Alosa fossiles répondent à la diagnose du genre ou bien présentent une ressemblance suffisante avec les espèces actuelles pour permettre une identification aisée, mais on ne peut attribuer ces fossiles, d'un point de vue phylogénétique, qu'aux clupéidés.

En tout cas, le rang générique attribué à ce groupe reste arbitraire. L'apparition d'une nomenclature phylogénétique, dont le code est en cours de rédaction et consultable sur Internet (http://www.ohiou.edu/phylocode/) mettra peut être fin à l'utilisation des rangs taxonomiques, et même à la nomenclature binominale, selon des modalités qui restent à définir.

\section{CONCLUSION}

Les aloses fossiles n'ont pas fait l'objet d'études autre que la description originale. La monophylie du genre n'a donc pas été testé. D'ailleurs, GRANDE (1985) n'a pas pu proposer une seule synapomorphie pour aucun groupe de Clupeidae. Pour cet auteur, c'est le principal problème phylogénétique qui reste à résoudre au sein des Clupeomorpha.

Les descriptions ne permettent pas toujours de proposer de caractères intéressants pour une analyse phylogénétique. Leur but est l'identification et non l'établissement des relations de parenté, mais cette distinction ne peut être faite que si l'on dispose d'une théorie explicite des classifications. Or les systématiciens tenants de la théorie synthétique n'ont jamais produit de méthodes.

La méthodologie cladiste permet de retracer la structure (pattern) des relations de parenté, indépendamment des mécanismes (process) qui les ont produites. Une conséquence de l'application des concepts phylogénétiques, avec ses critères de testabilité et de transparence méthodologique, a été la critique de la nomenclature linnéenne, créée dans un contexte fixiste. A partir des travaux de DE QUEIROZ et GAUTHIER (1990), une nomenclature phylogénétique, avec un «phyllocode » propre, commence à se dessiner.

Des études doivent être menées sur les aloses actuelles et fossiles, afin de permettre la proposition d'hypothèses phylogénétiques susceptibles de définir le contenu et les caractéristiques d'un éventuel taxon Alosa. Ces études auraient une importance certaine dans l'établissement des relations de parenté des sous-groupes de Clupeidae. Ces résultats pourraient être appliqués dans le cadre d'études écologiques, de conservation et de gestion, etc. En effet, la connaissance des relations de parenté permet d'inférer certaines informations sur les traits de vie pour des taxons moins étudiés et de maximiser l'efficacité des travaux des gestionnaires de la biodiversité.

En attendant ces études, on peut parler de douze espèces fossiles d'Alosa du point de vue de la nomenclature linnéenne. D'un point de vue phylogénétique, il n'y a pas de fossiles appartenant au genre Alosa, et les espèces fossiles attribuées à ce genre doivent être considérées comme des Clupeidae incertae sedis. La recommandation donnée par GRANDE (1985) est de mettre le nom de genre entre guillemets tant que la position systématique de ces taxons fossiles n'a pas fait l'objet d'une étude plus approfondie. 


\section{REMERCIEMENTS}

Je voudrais remercier J.L. BAGLINIÈRE de m'avoir invité à participer au colloque Aloses 2000. Le manuscrit a pu être amélioré grâce aux commentaires d'un relecteur anonyme.

\section{BIBLIOGRAPHIE}

ARAMBOURG C., 1927. Les poissons fossiles d'Oran. Matériel Cart. Geol. Algérie, 6, 5299.

BEATY J., 1982. Classes and cladists. Systematic Zoology, 31, 25-34.

BERTIN L., ARAMBOURG C., 1958. Super-ordre des Téléostéens. In : GRASSÉ P.P. Traité de Zoologie, 13 (3), 2204-2500.

DARLU P., TASSY P., 1993. La reconstruction phylogénétique : concepts et méthodes. Masson, Paris, $245 \mathrm{p}$.

DE QUEIROZ K., GAUTHIER J., 1990. Phylogeny as a central principle in taxonomy : phylogenetic definitions of taxon names. Syst. Zool., 39 (4), 307-322.

D'UDEKEM-GEVERS M., 1990. L'analyse cladistique, problèmes et solutions heuristiques informatisées. Biosystema, 4, 1-114.

ELDREDGE N., 1985. Unfinshed Synthesis. Biological Hierarchies and modern Evolutionary Thought (Trad. esp.). $284 \mathrm{p}$.

GRANDE L., 1985. Recent and fossil clupeomorph fishes with materials for revision of the subgroups of clupeoids. Bulletin of the American Museum of Natural History, 181, 231-372.

HENNIG W., 1966. Phylogenetic systematics. 233 p.

LE GALL O., 2000. L'origine et l'histoire des aloses. In : ELIE P., BAGLINIÈRE J.L., Les aloses en France. INRA-Cemagref.

NELSON G.J., 1978. Ontogeny, phylogeny, paleontology, and the biogenetic law. Syst. Zool., 27, 324-345.

PATTERSON C., 1982. Classes and cladists or individuals and evolution. Syst. Zool., 31, 284-286.

VON BAER K.E., 1828. Uber Entwicklungsgeschichte der Thiere. Beobahtung und Reflexion. Borntriger, Knigsberg.

WILEY E.O., 1976. The phylogeny and biogeography of fossil and recent gars (Actinopterygii : Lepisosteidae). Misc. Publ. Mus. Natur. Hist. Univ. Kansas, 64, 1111.

WHITEHEAD P.J.P., 1985. FAO species catalogue. Vol. 7. Clupeoid fishes of the world (Suborder Clupeoidei). Part 1. Chirocentridae, Clupeidae and Pristigasteridae. $303 \mathrm{p}$. 